\title{
La RSE entre Institution du capitalisme et stratégie productive : une grille de lecture
}

Catherine Bodet et Thomas Lamarche

\section{(2) OpenEdition \\ Journals}

Édition électronique

URL : http://journals.openedition.org/ei/5738

DOI : 10.4000/ei.5738

ISSN : 2553-1891

Éditeur

Association Économie et Institutions

Référence électronique

Catherine Bodet et Thomas Lamarche, «La RSE entre Institution du capitalisme et stratégie productive : une grille de lecture », Économie et institutions [En ligne], 24 | 2016, mis en ligne le 30 juin 2016, consulté le 05 mai 2019. URL : http://journals.openedition.org/ei/5738; DOI : 10.4000/ei.5738

Ce document a été généré automatiquement le 5 mai 2019.

Revue Économie et institutions 


\title{
La RSE entre Institution du capitalisme et stratégie productive : une grille de lecture
}

\author{
Catherine Bodet et Thomas Lamarche
}

1 La Responsabilité sociale de l'entreprise (RSE) est régulièrement présentée en tant que volet entreprise du développement durable ${ }^{1}$ : dans la période actuelle, elle vise à apporter à cette échelle des réponses à la crise écologique et sociale mondiale (cf. la périodisation de Capron et Petit, 2011). La notion RSE est donc conjointement utilisée pour qualifier les pratiques décentralisées et volontaires des entreprises, et au-delà pour caractériser une dynamique normative plus structurante. La RSE est ainsi constitutive d'innovations institutionnelles (Bodet et Lamarche, 2007) visant l'autoproduction de règles par les acteurs: les entreprises elles-mêmes (Capron et Quairel-Lanoizelée, 2004), mais aussi leurs représentants ${ }^{2}$. Ainsi la norme ISO 26000 relative à la responsabilité sociétale, dite aussi RSE/DD, renvoie-t-elle à l'action des entreprises en faveur du développement durable, sans recourir à la certification ni à labélisation.

2 Face à cette caractérisation qui a pour origine l'ambition de nombreuses grandes entreprises à une légitimation par la notion très plastique de développement durable, nous avons pour objet d'interroger en quoi la RSE peut être une contribution effective de l'entreprise au développement durable, sachant que le développement durable se situe à l'échelle macro. La question ainsi posée est la suivante: la RSE est-elle en mesure d'infléchir ou remettre en cause le mode de régulation actuel, qualifié de financiarisé ou néolibéral, et de former une logique de développement durable ? Cette question interroge de façon directe la capacité de la RSE à transformer la régulation du capitalisme. Nous considérerons une appréhension générale du développement durable, sans entrer sur la notion de décroissance notamment, et en considérant la notion dans son aspect très malléable issu des années 1980 (Martínez-Alier et al., 2010). En nous inscrivant dans la perspective de la théorie de la régulation (Boyer et Saillard, 2002) nous rejoignons sa problématique centrale qui est la caractérisation du régime dominant, en participant à la compréhension de la régulation définie comme la «conjonction des mécanismes 
concourant à la reproduction d'ensemble, compte tenu des structures économiques et des formes sociales en vigueur » (Boyer, 1986, p. 30). Nous questionnons la capacité de la RSE, en tant que volet entreprise du développement durable, à contribuer à une montée en régime des principes de responsabilité écologique et sociale. C'est dans ce sens que nous portons attention à la codification des rapports sociaux ${ }^{3}$ dans lesquels le niveau entreprise est chargé de prérogatives et de libertés élargies. Il ne s'agit pas pour autant de considérer que les pratiques microéconomiques forment d'elles-mêmes la montée en régime, toutefois la remise en cause d'institutions centrales du rapport salarial et la codification du rapport à l'environnement accordent aux entreprises plus d'autonomie (Rousseau et Zuindeau, 2007). Nous montrerons que la RSE s'inscrit ainsi en tant que processus de désinstitutionnalisation du rapport salarial par un recours accru à des dispositifs décentralisés (codes de conduite, chartes d'entreprise etc.)

Dans la régulation financiarisée, les acteurs dominants de la sphère de la production sont les Firmes Multinationales (FMN) (Michalet, 1985), et ce sont elles qui vont façonner et définir la notion de RSE et ses instruments. C'est pour cette raison que nous focalisons sur les FMN, sans présumer qu'il n'y a que cette catégorie d'entreprises qui utilise des dispositifs de $\mathrm{RSE}^{4}$. Ajoutons que l'on ne traitera pas de l'ensemble des responsabilités réelles des entreprises, mais de la RSE comme phénomène porté par les FMN.

4 L'objet essentiel de cet article est la proposition d'une grille de lecture synthétique orientée par les approches en termes de régulation (Boyer et Saillard, 2002). En effet, dans les travaux régulationnistes une attention particulière est portée à la construction de " faits stylisés ", initialement dans une filiation macroéconomique ouverte par Kaldor et perpétuée par Boyer qui les met au centre de l'approche régulationniste : «L'idéal serait de disposer d'une théorie, aussi simple que possible quant à ses hypothèses, logiquement cohérente et rendant compte de la totalité des faits stylisés sous revue» (Boyer, 1992) Comme dans toute construction de fait stylisé, nous opérons une réduction de spécificités. La discussion à propos de ce réductionnisme a été très largement nourrie en ce qui concerne notamment les faits stylisés qui ont caractérisé le fordisme. De façon similaire, afin de saisir les traits génériques de la RSE dans le capitalisme financiarisé, nous ne pouvons pas traiter des très grandes variétés observables. Nombre de travaux ont toutefois rendu compte des formes locales / nationales de la RSE, de même que des variétés sectorielles. La grille de lecture conceptuelle que nous proposons s'insère d'ailleurs dans une démarche mésoéconomique dont l'objet est précisément de rendre compte de la façon dont des espaces (secteurs, territoires, chaînes de valeur, entreprises, professions) se différencient et développent, par bricolages, une très grande variété de dispositifs institutionnels issus de l'action d'acteurs cherchant à défendre leurs intérêts (Lamarche et al., 2015).

5 La proposition est donc essentiellement analytique et non-empirique, quoi qu'elle soit nourrie de différents terrains et de l'étude de pratiques de nombreux groupes ${ }^{6}$. Afin de comprendre comment agit la RSE, nous proposons ainsi une grille de lecture basée sur deux faits stylisés, qui sont deux enjeux centraux de régulation et de pouvoir. L'un est de nature institutionnelle / politique (nous le désignons RSE-Institution). Il rend possible et stabilise le second (RSE-Stratégie) de nature économique / productive qui en retour engendre et requiert des compromis politiques que structurent les dispositifs institutionnels. La RSE-Institution réfère à la capacité des dispositifs institutionnels de la RSE à donner corps à une logique politique conduisant un compromis social (au sens des « compromis sociaux institutionnalisés » définis par André et Delorme, 1983). La RSE- 
Institution produit et soutient des compromis qui sont toutefois faibles, car réversibles et asymétriques. Ils ne sont ainsi pas à même de modifier structurellement la régulation financiarisée, mais en infléchissent toutefois les dispositifs institutionnels (Partie 1). La RSE-Institution en engendrant de nouveaux dispositifs et de nouvelles règles donne un cadre à une évolution de la production, soutenant l'émergence de ce que nous caractérisons en termes de RSE-Stratégie. La RSE-Stratégie est ainsi basée sur des logiques productives basées sur des principes de RSE, alliant la construction de stratégies de profit et la recherche d'avantages concurrentiels, dans l'esprit de ce que décrivent Porter et Kramer (2006). Cette dynamique productive repose sur la création par les acteurs d'une pluralité de conventions de qualité en termes de responsabilité qui sont toutefois de portées inégales et limitées, mais sont des signaux importants d'évolution des marchés de biens et des services. La RSE-Stratégie se développe en engendrant de nouveaux instruments de gouvernance interfirme, particulièrement au sein des chaînes globales de valeur (CGV), soutenus par les dispositifs que porte la RSE-Institution. Nous montrerons qu'elle ne permet pas toutefois, en l'état, un dépassement du régime financiarisé et tend, au contraire et de façon inattendue, à favoriser son renforcement, tout en engendrant toutefois des pratiques nouvelles dans la production (Partie 2).

6 Montrer comment joue l'interaction entre les dispositifs institutionnels portés par la RSEInstitution et la dynamique de production de la RSE-Stratégie permet de saisir la régulation des contraintes dans laquelle la RSE est mobilisée, sans être nécessairement décisive. La double dimension politique / institutionnelle d'une part, stratégique / productive de l'autre, se comprend dans un rapport dialectique entre ces deux construits qui ne sont pas des objets différents, mais deux grandes fonctions ou natures de la RSE : institution d'une part, stratégie économique de l'autre (cf. Schéma).

7 Nous montrons au final que si la RSE-Institution n'a que peu d'influence sur les rapports sociaux fondamentaux, n'infléchissant pas la régulation d'ensemble, elle est susceptible de donner forme à une RSE-Stratégie qui fait évoluer en retour les rapports de production. Toutefois, les stratégies et les institutions de la RSE sont inscrites dans une régulation oligopolistique privée qui s'est imposée à la fin du $20^{\mathrm{e}}$ siècle, et rien ne signale leur capacité à agir dans le sens d'un développement durable dans son acception générale et systémique. 


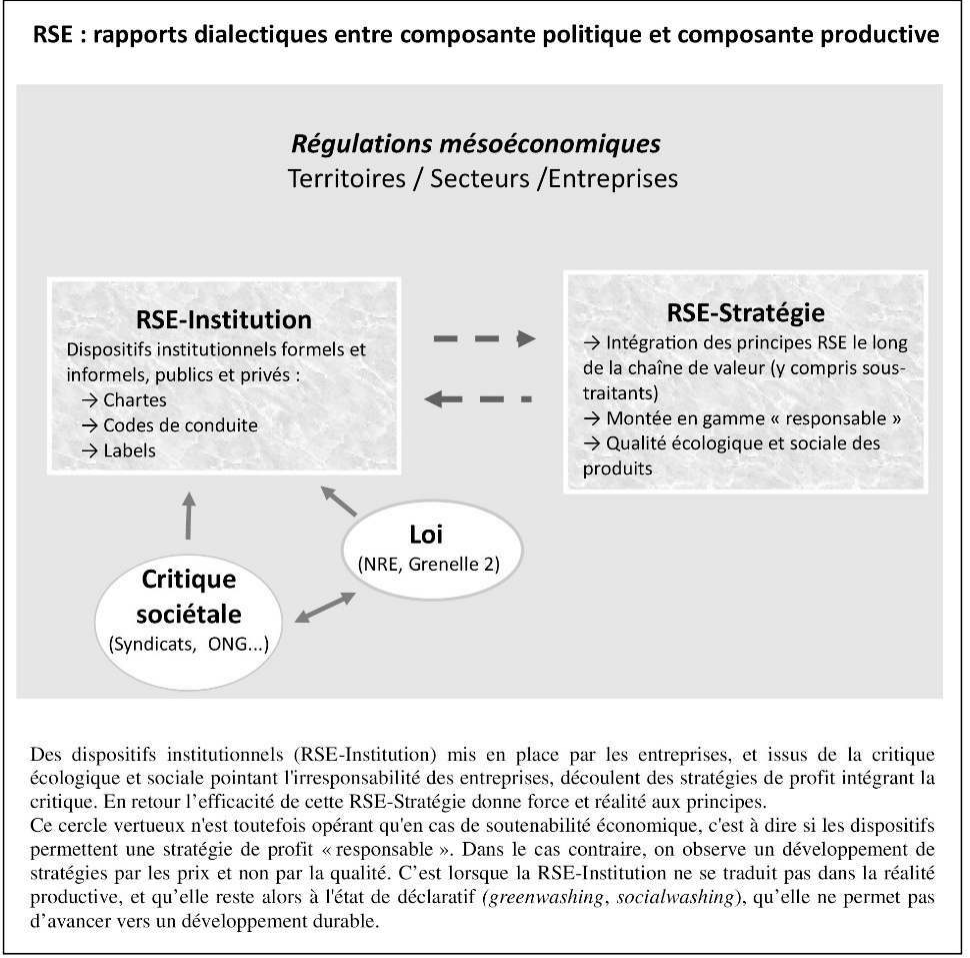

\section{RSE-institution : Reproduction des pouvoirs du régime financiarisé}

8 La dynamique de RSE est constituée, voire définie, par les dispositifs institutionnels qui sont engendrés en son nom et procède en tant qu'enjeu de nature politique. Ainsi la définition de la RSE qui part de l'échelle des entreprises et des relais qui poursuivent son action (ONG inféodées, réseaux d'influence, lobbies) est en soi un enjeu de pouvoir sur l'édiction des règles qui structurent l'action. La RSE-institution suit le chaotique processus de définition de la RSE énoncée par de nombreuses institutions internationales, en ce sens, le processus d'institutionnalisation de la RSE est «un foisonnement perturbant mais signifiant des rapports de pouvoir économique » (Chanteau, 2011). En tant qu'institution, la RSE nous apparaît en tant que nécessité politique du régime financiarisé, elle assure une fonction de légitimation, notamment par un processus de récupération de la critique (1.1). Les dispositifs institutionnels mis en œuvre sont intrinsèquement liés à la structure de la firme mondialisée et à la dilution de sa responsabilité formelle (1.2) ce qui souligne les limites des rapports de force susceptibles de modérer le pouvoir de la finance, et de modifier les formes de concurrence qui agissent à l'échelle globale.

\subsection{La RSE, nécessité politique du régime financiarisé}

Durant les années 1990 les travaux régulationnistes ont montré que le régime de croissance était financiarisé, soulignant la domination de la sphère financière et sa 
capacité à s'approprier la valeur7. Aglietta (1998) caractérise un « régime de croissance patrimonial », qui émerge alors dans un seul pays et s'exerce grâce à une domination proprement financière, assurée par les instruments et dispositifs de la finance. Lordon (1999) identifie pour sa part un "régime d'accumulation financiarisé» qui coexiste cependant avec une pluralité de modes de régulation. La domination de la finance sur le régime d'accumulation se traduit par différentes réponses politiques permettant la reproduction des rapports sociaux fondamentaux. En dépit de crises récurrentes depuis 1987, et de la grande crise qui débute en 2007 , le régime perdure toutefois, du fait du caractère fortement institué des instruments financiers. On utilisera par la suite la notion de régime financiarisé en associant la dynamique propre du régime d'accumulation aux mécanismes qui assurent sa reproduction, que les approches en termes de régulation placent sous le terme de mode de régulation.

10 La RSE-Institution prend place dans la dynamique déréglementaire propre à la régulation financiarisée, remettant en cause les mécanismes et compromis fondateurs du fordisme : libéralisation des marchés financiers, flexibilisation du travail, privatisation des services publics, remise en cause de la protection sociale, désinflation compétitive. La RSEInstitution provient d'une réaction face au développement de la critique sociale et écologique au moment où il devient politiquement nécessaire de développer une réponse aux dégradations perceptibles du point de vue social et environnemental. La RSEInstitution a les attributs de la période déréglementaire et du primat de la firme mondialisée; elle procède selon une logique d'autoréglementation, qui est la traduction de la décentralisation de la production institutionnelle à l'échelle des entreprises. En ce sens, la RSE procède à bonne distance de la règle publique, légale et commune, pour privilégier un discours d'engagement privé, volontaire et non contraignant. Au final, c'est quand la firme n'est plus considérée comme une institution au service des stakeholders (Roger et al., 2012) que la RSE-Institution émerge, produisant un discours de nature politique et engendrant des règles peu contraignantes.

\section{Récupération de la critique sociétale : les origines défensives de la RSE}

11 À l'origine de la RSE contemporaine, dite de soutenabilité, se trouvent des démarches défensives, de réponse à la critique sociétale exprimée par différents groupes sociaux face aux « irresponsabilités » économiques et sociales des entreprises (Descolonges et Saincy, 2004). Les réactions défensives sont le point de départ tant à l'échelle d'entreprises qui, prises en défaut quant à leurs pratiques, sont mises en situation de se défendre afin de préserver leur capital réputationnel, qu'à l'échelle des entreprises en tant que collectif défendant leurs intérêts communs. Des collectifs entrepreneuriaux se constituent en effet en tant que blocs d'acteurs, notamment relayés à l'échelle sectorielle par des organisations professionnelles (Acquier et Aggeri, 2008). Ils ont comme intérêt commun de limiter la réaction réglementaire susceptible d'être portée par l'État à l'échelle nationale et de valoriser l'action des entreprises.

Ces démarches constituent les réponses directes aux demandes que portent des acteurs situés hors de l'entreprise, notamment des ONG qui constituent ce qu'une vision optimiste considère en tant que nouveaux stakeholders et une vision sceptique « cheval de Troie de la mondialisation néolibérale "(Barnejee, 2011) ${ }^{8}$. Les réactions défensives correspondent à une dynamique de justification et de légitimation dans le cadre d'un régime inégalitaire qui a réduit la justice sociale auparavant portée par l'État social et le 
rapport salarial fordiste. Elle procède par événement/accident selon une longue chronologie depuis le controversé sabordage de la plate-forme Brent Star propriété de Shell en Mer du Nord (1995) ${ }^{9}$ que dénonça Greenpeace, jusqu'à l'effondrement de l'immeuble Rana Plaza de Dacca au printemps $2013^{10}$.

La dynamique de RSE relève ainsi d'une structuration par évènements qui font figure de crises à l'échelle des firmes. Bien que traitant de cette échelle nous retrouvons l'idée d'Ernest Labrousse (1944) telle que « les économies ont les crises de leurs structures », la crise sert de révélateur permettant de caractériser ce qui fonde la régulation ${ }^{11}$.

La dynamique de communication est un moment clé de la diffusion d'une culture de la RSE (de la Broise et Lamarche, 2006). Les réactions défensives se traduisent par des « engagements " à éviter un nouvel accident. La traduction effective dans les lieux et structures de production (Partie 2) est toutefois bien moins forte que leur traduction dans des règles internes qui sont ainsi autant de dispositifs institutionnels faibles. Cependant l'événement-crise, et son effet potentiel en termes de perte de capital-marque et de marché, assure une disciplinarisation par la RSE. La littérature économique standard retient d'ailleurs, dans une acception stratégique, le fait que la RSE est devenue un instrument de discipline de marché, faisant comme si les déclarations et règles internes assuraient une discipline effective.

\section{ONG : extension du champ de la critique}

Le processus d'institutionnalisation de la RSE provient ainsi de démarches défensives et a pour vocation de répondre à des interpellations publiques. Boltanski et Chiapello (1999) ont montré le rôle central que joue la critique, concluant que le capitalisme doit sa longévité à sa capacité à la prendre en compte et l'assimiler. En l'occurrence, on assiste à une centralité d'une critique écologique, construite par combinaison de ce que les auteurs ont désigné sous le nom de critique artiste et de critique conservatrice, associées à une transformation de la critique sociale qui dominait dans la période fordiste. Le succès de la critique écologique (Chiapello, 2013) provient de sa capacité à saisir les médias et le politique via des stratégies médiatiques. Une critique conservatrice, plus ancienne, se situe dans la filiation du paternalisme et nourrit toutefois la thématique de la RSE par l'intermédiaire de l'éthique du manager et de la morale de l'entreprise présente notamment en Amérique du Nord (les fondations d'entreprise en sont une déclinaison).

Cette critique converge avec certains aspects de la critique artiste, notamment la critique de la société de consommation, la recherche d'authenticité, une demande d'épanouissement professionnel et d'autonomie, ainsi qu'avec un renouveau de la critique sociale, orientée vers les conditions d'exploitation du travail dans des pays du Sud, les droits humains et l'exacerbation des inégalités. Selon Chiapello, la critique écologique est un facteur de construction des normes du capitalisme en le forçant à incorporer des valeurs qui ont servi à le critiquer. La RSE assure cette fonction à propos de l'action des entreprises et participe d'un mouvement de légitimation de l'entreprise vis-à-vis de la société. Ce faisant, elle conduit les entreprises à élargir le champ de leurs prérogatives en endossant une responsabilité environnementale qui s'ajoute à leur « responsabilité » vis-à-vis des actionnaires ${ }^{12}$.

17 Le développement de la critique écologique en écho à des événements-crise se traduit dans la RSE-Institution qui est une codification de ce nouveau rapport social dominé par les grandes entreprises. Une erreur d'interprétation courante est toutefois de considérer 
que la RSE se situe exclusivement à l'échelle de l'entreprise. Or, c'est le propre d'un processus d'institutionnalisation de voir la normalisation échapper à l'action microéconomique pour devenir collectif et structurant.

\section{Co-construction des cadres réglementaires : de la capacité à agir sur les normes} question pour renégocier certains rapports sociaux dans lesquels le fordisme les avait mis en position de faiblesse et que la régulation financiarisée rend malléables, principalement le rapport salarial et les formes de concurrence, mais aussi l'exercice de l'autorité publique qu'assure l'État.

19 Il en résulte une instrumentalisation de la RSE dans le droit fil de la délégitimation de l'autorité publique (state failures) par différents groupes (entreprises, mais aussi think $\operatorname{tank}^{13}$ ) qui affirment que la prise en charge des externalités au niveau de l'entreprise est plus pertinente que leur administration publique.

La reconnaissance du primat de l'entreprise pour la définition de ses propres «responsabilités » est un point d'inflexion par rapport à la période fordiste durant laquelle l'État social s'est trouvé en charge d'un ensemble très étendu de responsabilités sociales (même si nombre d'entre elles sont assurées à l'échelle des entreprises). Ainsi, lorsque la Commission de l'Union européenne adopte en 2006 une communication dans laquelle elle déclare que la responsabilité sociale des entreprises relève " exclusivement " d'une approche volontaire et s'en remet aux seuls milieux d'affaires (Capron, 2006), il s'agit d'un net repli de l'autorité publique et d'une institutionnalisation de la capacité des entreprises à s'autoréglementer. Pour autant cela ne fonde pas une autorégulation, dans le sens où une décision publique, quelle qu'elle soit, ne transforme pas les rapports sociaux fondamentaux, et n'empêche pas les autres acteurs (État, partenaires sociaux, ONG) de rester partie intégrante de ces rapports sociaux.

21 Le rôle des entreprises est illustré au sein même des processus juridiques. En France, deux textes légaux ont été adoptés, la loi Nouvelles Régulations Economiques en 2001 et l'article 225 de loi Grenelle 2 en 2010. Pour ces deux cas, l'activité des entreprises pour coécrire les textes est symptomatique de leur motivation à limiter la portée de la loi. En l'occurrence elles s'opposent à l'accroissement du nombre des entreprises concernées par l'obligation de reddition, mais aussi à l'extension du champ des informations obligatoires et enfin au mode de contrôle mis en œuvre (Clos, 2013). Le délai entre la loi Grenelle 2 et le projet de décret (2010) et la parution du décret (2012, à la veille de l'élection présidentielle) est le signe de la tension entre l'État et l'action relayée des entreprises. La possibilité finalement accordée aux entreprises de substituer l'adhésion au dispositif EMAS (Eco Management and Audit Scheme) ${ }^{14}$ à l'obligation de contrôle du reporting qui est inscrite dans la loi montre que la rédaction finale du texte transforme le fond de l'intention énoncée. Le dispositif légal s'est ainsi déplacé depuis une logique de contrôle et de vérification dument inscrite dans la loi Grenelle 2 à un simple dispositif d'accompagnement des entreprises ${ }^{15}$. On a là un exemple type de la faiblesse de la RSEInstitution et de la volonté du patronat français de réduire le champ de la contrainte.

Dans le même esprit, le processus normatif qui conduit à l'adoption de la norme ISO 26000 en 2010 est exemplaire du niveau d'investissement des acteurs afin de produire leur propre convention de qualité ; c'est ce qui en fait d'ailleurs une "norme hors-norme " (Capron et al. 2011). Il ne s'agit donc pas seulement d'un processus privé (politique 
d'entreprise en matière de développement durable, de responsabilité...), mais plus fondamentalement d'un processus collectif impliquant la création de dispositifs communs : norme ISO, référentiels sectoriels, dispositif de formation, procédures, outils de gestion, émergence d'une profession ${ }^{16}$. En tant que définition non coercitive du contour, des règles et des obligations en matière de responsabilité sociétale, la RSEInstitution constitue un investissement collectif, immatériel. En effet ces dispositifs limitent la coercition en défendant à chaque étape légale le caractère volontaire de la RSE.

La RSE-Institution est à considérer en tant que ressource immatérielle partagée entre acteurs d'un même bloc de pouvoir. Dans le cas d'ISO 26000 la production de la norme ne se résume ni à un affrontement concurrentiel intermédié par la construction de dispositifs, ni à une forme de collusion des entreprises contre certaines parties prenantes, bien que le groupe « entreprises » ait été omniprésent lors des négociations (Capron et al. 2011). La normalisation ISO 26000 en tant qu'innovation institutionnelle est mobilisée par des acteurs, et en retour elle impose un cadre aux relations entre eux ${ }^{17}$. Un effet attendu de ces dispositifs est de centrer l'évaluation de la responsabilité sur un processus interne. Sont ainsi créées les conditions de la décentralisation de la reddition à l'échelle de l'entreprise.

\subsection{Firme mondialisée et dilution de la responsabilité formelle}

L'organisation de la production mondialisée transforme le périmètre de l'entreprise et opère une dilution de sa responsabilité formelle. La rupture de la fin du siècle est celle du passage de la mondialisation de la circulation à la mondialisation de la production, d'où l'importance des relations interfirmes. L'étalement dans les CGV (Gereffi et al., 2005), et l'imbrication via des filiales de nature variée assure conjointement la mise à distance (i.e. la non-propriété assurant la réversibilité) et le contrôle de la production (via le contrat). La gouvernance de la production est ainsi basée sur le couple autonomisation formelle (faire-faire) et responsabilisation économique des sous-traitants. Les changements affectant la grande entreprise, conduisant d'une dynamique de multinationalisation à la constitution de firmes financiarisées (1), se doublent d'une absence de définition juridique de l'entreprise qui dilue sa responsabilité (2). Dans ce cadre, le développement de la RSE-Institution ne transforme pas radicalement les formes institutionnelles de la régulation financiarisée (3).

\section{De la multinationalisation à la firme financiarisée}

Le « capitalisme mondial » (Michalet, 1985) qui se développe dans le dernier quart du 20é siècle est issu d'un triple basculement qui aboutit à placer les FMN dans une position centrale. Les deux premiers basculements correspondent au passage des échanges internationaux de biens et services à l'internationalisation de la production et donc à la multinationalisation des firmes. Alors, les firmes mondialisées, en internalisant les disparités nationales, parviennent à occuper une place clé dans la mondialisation de la production.

La régulation mondialisée est ainsi oligopolistique et dominée par les grandes firmes avant même la période de globalisation financière. La puissance des firmes multinationales ne se situe plus essentiellement dans le cadre du commerce 
international, car les activités à l'étranger et les échanges intrafirmes supplantent la dimension commerciale. Elles dominent hiérarchiquement leur chaîne de producteurs de par leur position oligopolistique. La relation de subordination qui s'instaure avec un certain nombre de pays n'est toutefois pas unilatérale, l'action des États sur les firmes dépendant des ressources dont disposent les pays (et notamment des ressources immatérielles, telles les compétences, le niveau de technologie, mais aussi la stabilité politique ${ }^{18}$ ). Dans ce contexte, la RSE est un instrument mobilisé contre certains dispositifs de l'Etat social: non seulement contre les réglementations contraignantes, mais aussi contre les compromis sociaux ${ }^{19}$.

Avec la montée en puissance du régime financiarisé, les firmes sont elles-mêmes mises sous pression de la finance de marché afin d'engendrer des taux élevés de rendement du capital. Ainsi, le report de la contrainte sur les fournisseurs s'accélère.

La concentration croissante du capital financier auprès des investisseurs institutionnels, gestionnaires de l'épargne collective, structure le pouvoir managérial. De nouveaux critères d'efficience ont été spécifiés au profit de la puissance retrouvée des actionnaires. Les acteurs dominants sont désormais les FMN et les investisseurs institutionnels qui agissent à l'échelle de marchés financiers mondialisés. La conception actionnariale de l'entreprise (Fligstein, 1990) s'étend à tous les secteurs du fait de la puissance des dispositifs institutionnels de financiarisation. Ces acteurs internationaux tendent ainsi à instaurer une régulation oligopolistique privée (Michalet, 1985). Etendue à l'échelle mondiale cette régulation est le terreau du développement de la RSE-Institution. C'est à ce niveau, peu normé compte tenu de la faiblesse politique de la gouvernance mondiale, que les FMN vont penser des stratégies institutionnelles via la RSE.

La dynamique de production de règles privées qu'impulsent les FMN, atteste de la capacité de certains acteurs multinationaux d'infléchir, voire de construire les dispositifs institutionnels. Les acteurs dominants mobilisent en effet les règles juridiques et façonnent les normes (Jeammaud, 2000), qu'elles soient à caractère général et public ou privé. Ces stratégies institutionnelles débouchent sur des propositions de soft Law, les entreprises procédant par évitement des processus législatifs en la matière, notamment en produisant leurs propres corps de règles privées qui prennent sens dans la RSEStratégie.

\section{De l'absence de définition juridique de l'entreprise à la dilution de sa responsabilité}

30 La responsabilité juridique de l'entreprise et notamment de l'entreprise multinationale pose d'importantes difficultés car il n'y a pas de définition de la FMN en droit, ni, en droit français, de définition de la notion d'entreprise, par opposition à celle de société. En anglais se trouve la même tension entre enterprise et firm d'une part qui ne sont pas définies juridiquement, et corporation et company, d'autre part, qui le sont. L'entreprise, comme le rappelle Favereau (2012, p.23), est une «organisation économique, sans existence juridique officielle, dont la composition des membres est indéterminée...». Ainsi la société est un sujet de droit, c'est une entité juridique, alors que l'entreprise est une organisation économique, qui rassemble de multiples composantes, voire différentes sociétés.

31 Les FMN plus encore que les autres entreprises ont la capacité de définir leurs frontières légales par l'intermédiaire de la structure de leur capital, en l'occurrence en définissant les frontières entre les sociétés juridiquement constituées (notamment à l'échelle 
internationale). D'un point de vue formel, juridique, la coupure entre la société de droit et l'entreprise comme entité économique non définie en droit, dégage la société de ses responsabilités hors de sa définition en droit. Dès lors que la firme mondialisée n'exerce pas un pouvoir de droit, elle est à même de décliner sa responsabilité de droit. S'appuyant sur un argumentaire juridique et économique, Favereau et Robé (2012) caractérisent la " grande déformation » de l'entreprise, et s'attachent, à la suite de Blair (2000), à montrer le rôle structurant de la théorie de l'agence dans la restauration du pouvoir des actionnaires. En donnant un cadre à la fiction de la propriété sur l'entreprise, la théorie de l'agence efface la distinction entreprise / société et confond droit de propriété sur les titres (actionnaires, propriétaires de titres) et droit de propriété sur l'entreprise ellemême. La théorie de l'agence opère comme s'il y avait une transitivité dans la propriété : le propriétaire des titres d'une société serait propriétaire des actifs de cette personne morale. Or les propriétaires des titres, s'ils sont loin d'être sans pouvoir, ne sont pas propriétaires de l'entreprise. En l'occurrence, les propriétaires des titres ne sont pas responsables juridiquement de l'entreprise. Entre ces deux niveaux de propriété, l'un avéré (propriété des titres) et l'autre proclamé (propriété sur l'entreprise), se situe un enjeu en termes de pouvoir.

32 Le pouvoir que l'actionnaire exerce ne se réduit pas au droit, il utilise le droit, mais se traduit dans quantité d'autres instruments de pouvoir: subordination, domination symbolique, instauration de normes... ainsi que capacité d'échapper à la responsabilité juridique. On peut conclure qu'« il n'y a pas de pouvoir de jure (fondé sur la propriété) dans le gouvernement externe de la firme-monde (...) En d'autres termes seul demeure le pouvoir de facto " (Chassagnon, 2013).

33 Le développement des FMN, dans la phase de multinationalisation de la production, agit par dissociation des frontières légales et du périmètre économique de l'entreprise. Dans ce sens les compromis institutionnalisés, notamment à propos du travail et du droit du travail sont contournés. Alors, puisque la firme choisit sa taille et conjointement les frontières de sa responsabilité juridique, elle choisit de ce fait les limites de ses responsabilités légales, fournissant une invitation à l'évitement de ses devoirs légaux obligatoires (Collins, 1990). Les dispositifs institutionnels sont conditionnés par une démarche stratégique de production, qui consiste à organiser l'allocation multinationale des ressources en diminuant la responsabilité formelle. Le développement de la RSE prend place dans cette interaction entre motifs institutionnels et dynamique économique. Alors que les emboîtements dans la chaîne de valeur constituent un évitement de la responsabilité et un report sur les fournisseurs, la dynamique de la RSE s'empare progressivement de cette problématique et forme le nœud d'un nouveau rapport de force qui s'exprime dans la création de dispositifs de soft law (charte, guide, code de conduite, cahier des charges), mais ne s'exprime que faiblement en comparaison du pouvoir institué de la société de droit donneuse d'ordres.

34 Parmi les dispositifs de la RSE-Institution, rien ne remet donc en question la conception actionnariale de la firme (shareholder governance, selon Jensen et Meckling) qui s'est imposée face à la conception managériale (managerial capitalism, selon Berle et Means ou managerial class selon Chandler). La firme pensée à partir du nœud de contrat et de la relation d'agence a produit une fiction légale, adaptée à la période néolibérale qui remet en cause la firme managériale conçue comme espace collectif d'accumulation de compétences et gérée comme une entité instituée (Weinstein, 2012). La question centrale de la relation entre managers et actionnaires rejoint l'analyse de Duménil et Levy (2011) 
selon lesquels la classe managériale est au cœur du compromis avec les actionnaires et a permis la restauration de leur pouvoir. La financiarisation de la rémunération des dirigeants est un des dispositifs clé de ce compromis, elle aligne les incitations (et les désirs) des managers sur les intérêts de la finance de marché. C'est à l'aune de ce type de dispositifs que l'on peut mesurer de quelle façon la définition même de l'entreprise reste fondamentalement celle de la régulation financiarisée. Les dispositifs propres à la RSEInstitution sont de ce point de vue inopérant à s'opposer au rendement de la finance et aux bénéfices qu'en tire le groupe bien spécifique des hauts dirigeants (directors) en termes de pouvoir sur l'entreprise, et en termes de rémunération (Boyer, 2005).

La question de fonds d'investissement et de la "démocratie actionnariale " illustre la tension entre évolution de la régulation endogène et modification structurelle de la régulation. Si Aglietta (1998), considère que les salariés ainsi que les syndicats via la gestion de l'épargne salariale sont à même de réduire l'asymétrie d'information vis-à-vis des actionnaires et de porter un développement de long terme, de nombreux auteurs ont considéré que la norme de rendement dans laquelle agissent ces fonds empêchait structuralement de faire évoluer le rapport financier. Les normes de rendement suppriment leur marge de manœuvre et s'imposent comme règles pour le secteur. Une innovation financière de ce type est a priori favorable aux salariés, elle est typique de la RSE-Institution, mais ne permet en rien de sortir des normes et de l'incertitude radicale de la finance ${ }^{20}$. Ainsi, les professionnels de la finance qui gèrent ces fonds opèrent de la même façon, quel que soit le donneur d'ordres, demandant les mêmes rendements que la finance de marché (Plihon, 2001).

\section{La RSE-Institution ne transforme pas les formes institutionnelles}

La RSE-Institution est issue de l'action décentralisée des acteurs, et fait intervenir de nouvelles catégories (ONG, organismes internationaux, gate-keepers ${ }^{21}$ ). Mais avec la remise en cause des prérogatives publiques des États (y compris sur le plan des politiques économiques et sur la réglementation du travail, comme en atteste la situation européenne), la hiérarchie entre ces acteurs renforce la position des FMN.

La RSE-Institution se développe en tant que nécessité politique dans la régulation financiarisée, selon un processus de légitimation. Il s'agit d'un enjeu politique de régulation des pouvoirs et d'édiction de principes et de règles qui régissent la société : il s'agit de définir la responsabilité des acteurs et de fixer des normes de justice (sociale, environnementale). La RSE-Institution engendre des dispositifs de régulation qui se réfèrent à l'intérêt général et affirment une logique de développement durable, pourtant, elle n'engendre pas de rupture avec la régulation financiarisée, elle correspond en ce sens à une "régulation endogène " (Hoang Ngoc et Tinel, 2005). En mettant en œuvre des dispositifs supplémentaires de sélection endogène, les institutions de la RSE assurent une fonction (macro-institutionnelle) au sein de la régulation financiarisée. De la même façon que l'évolution du capitalisme est jalonnée d'innovations qui permettent sa reproduction, la RSE participe de ces innovations institutionnelles (Bodet et Lamarche, 2007) qui permettent la reproduction du capitalisme financiarisé, sans en être un moteur principal. La RSE-Institution, comme d'un autre point de vue certaines innovations techniques (TIC par exemple), renforce la division internationale du travail et la mondialisation des formes de concurrence et participent à leur codification. 

collectives, est de penser des dispositifs qui n'ont pas pour seul objet de reproduire l'ordre établi, mais de l'amender; il s'agit là de la dimension normative implicite qui suit la construction des faits stylisés. La pensée régulationniste s'est en effet focalisée sur la compréhension de ce qui permet à une économie donnée de perdurer (reproduction), alors même que les rapports sociaux conflictuels pourraient produire une instabilité permanente. En cela elle renoue avec la question fondamentale de l'économie politique que pose Smith: comment expliquer que la poursuite par chacun de son intérêt particulier ne conduit pas au chaos? La théorie de la régulation ne traite pas d'équilibre, mais des compromis sociaux temporaires qui permettent de réguler les tensions et contradictions, elle procède ainsi par analyse structurale du capitalisme, le mode de régulation étant ainsi défini par un "ensemble de procédures et de comportements individuels et collectifs qui a la triple propriété de: reproduire les rapports sociaux fondamentaux à travers la conjonction de formes institutionnelles historiquement déterminées; soutenir et piloter le régime d'accumulation en vigueur; assurer la compatibilité dynamique d'un ensemble de décisions décentralisées » (Boyer, 2004, p. 54).

Les conditions d'émergence et l'instrumentalisation des dispositifs institutionnels de la RSE montrent par la façon même dont ils sont socialement situés, qu'ils contribuent à la reproduction du modèle dominant. Cependant ils procèdent d'un rapport social qui évolue en permanence et arment certains acteurs, notamment des acteurs nouvellement impliqués (particulièrement les ONG et l'activisme citoyen). Ainsi ces dispositifs ne relèvent pas d'une reproduction à l'identique de la régulation néolibérale. La notion de reproduction sociale ne recouvre en rien une reproduction à l'identique. Ce sont les faits stylisés, formulés ex-post, qui construisent une représentation de la reproduction. En l'état actuel les dispositifs de la RSE tendent à reproduire l'ordre établi. Pourtant, la nature même d'un dispositif institutionnel est de modifier un rapport de force, et de ce fait la RSE-Institution n'est pas seulement un instrument de reproduction.

Les événements-crises associés à la RSE agissent comme des petites crises, faisant évoluer la régulation financiarisée. Elles sont le symptôme de dysfonctionnements structurels (en termes de répartition et de justice sociale, de destruction écologique). La capacité de récupération de la critique et l'institutionnalisation du régime parviennent à absorber les chocs comme autant de crises conjoncturelles, reproduisant le régime, et accroissant son instabilité. Si la capacité de la RSE à infléchir le régime est faible dans le sens où la RSEInstitution n'affecte pas les rapports sociaux fondamentaux, sa capacité à infléchir les stratégies productives est cependant une question essentielle pour engendrer des progrès sociaux.

\section{RSE-Stratégique : vers une intégration au sein des stratégies productives?}

41 La traduction de la RSE depuis le champ politique vers celui de la production et de l'organisation productive a pour corolaire de nouveaux compromis et de nouvelles tensions entre les différents centres d'accumulation. Cependant ces transformations sont partielles, souvent non prévues, les dispositifs de RSE peinent à produire les effets annoncés : ils restent marqués par une logique de légitimation. La RSE-Stratégie est une concrétisation au sein de la sphère de la production des règles portées par la RSE- 
Institution; c'est ce qui conditionne la montée en régime de la RSE, et qui reste hypothétique à l'heure actuelle. La RSE-Stratégie ne peut en effet s'imposer que si elle assure une rentabilité. Or le très haut niveau de rentabilité du capital est une caractéristique centrale du régime d'accumulation financiarisé et engendre d'ailleurs son insoutenabilité politique (Dannreuther et Petit, 2012). La RSE-Stratégie ne s'imposera donc que si elle remplit ces obligations de rendement, sauf à envisager une dynamique qui échappe à la financiarisation. En ce sens, il y a une opportunité de restauration d'une firme managériale portant une RSE-Stratégie lui permettant ainsi reprendre pied au sein de la régulation oligopolistique.

Dans la période actuelle, les dispositifs RSE sont des instruments de pouvoir interfirme au sein des CGV (2.1). L'émergence de nouvelles conventions de qualité est contrariée par un régime de demande incompatible et des formes de concurrence qui contournent les rapports salariaux nationaux. Ainsi les conditions de possibilité d'un nouveau régime d'accumulation ne sont pas réunies (2.2).

\subsection{Les dispositifs RSE comme instrument de pouvoir interfirme : les chaînes globales de valeur}

43 La structuration en réseau d'entreprises n'induit pas en tant que telle une gouvernance partenariale, contrairement à ce que la théorie des parties prenantes, qui est une référence clé de la RSE, l'indique (Dupuis, 2008). Nous assistons au contraire à une légitimation par les dispositifs RSE de la domination des FMN sur leurs fournisseurs, et à un report de la responsabilité sur ceux-ci, ainsi que sur les salariés. L'enjeu clé de la RSE réside dans l'articulation entre le registre politique (les intentions et les règles) et le registre productif (traduction de ces règles en termes de valorisation économique) (cf. Schéma).

\section{Une opportunité d'inflexion de la domination dans les chaînes de valeur?}

Le tassement des gains de productivité dans les années 1970 dans les pays de l'OCDE, puis la dynamique de mondialisation du capital et les politiques néolibérales de partage de la valeur qui ont suivi à partir des années 1980-1990, ont poussé les entreprises à investir la notion de la RSE, d'une part pour chercher de nouvelles niches, et d'autre part pour faire face à la crise du productivisme (Chanteau et Lamarche, 2013). La construction d'avantages concurrentiels est ainsi envisagée par différenciation et non plus tant par économies d'échelle et de variété, ce qui conduit à cibler différentes niches de marché susceptibles de faire remonter le taux de marge. La période qui prévaut dans les années 1980 et 1990 est ainsi celle d'une RSE « utilitariste » durant laquelle les efforts sociétaux ou environnementaux sont conditionnés au résultat économique de l'entreprise. Si l'entreprise développe des objectifs extra-économiques, c'est in fine, le rendement financier sur l'investissement qui mesure l'efficacité.

Cependant, en dépit d'un grand nombre d'études par méta-analyse (Margolis et Elfenbein, 2008, Orlitzki, et al., 2003) il n'a jamais été possible de déterminer de façon économétrique un effet unique des politiques RSE sur la performance financière, qu'il soit positif ou négatif. L'incertitude de ces études, et notamment la variété liée aux différentes situations a cependant favorisé une lecture validant la RSE, dans le sens où, elle n'induit pas d'effet négatif. Deux explications antagonistes perdurent et sont mobilisées : la RSE 
reste une approche communicationnelle et ne limite ainsi pas les performances financières de l'entreprise ; la RSE permet d'accroître la valeur de ses actifs intangibles, en renforçant ses droits de propriété ou en répondant à la critique écologique par la production de preuves (indicateurs extra-financiers, bilan GES (gaz à effet de serre), rapports de développement durable, etc.) (Lamarche et Rubinstein, 2012).

Cette conception utilitariste recoupe la notion de «RSE offensive » que définissent Porter et Kramer (2006) en lien avec le degré de contrôle de la chaîne de valeur. La RSE-Stratégie devient alors un attribut du pouvoir de la grande firme dans la globalisation, alors qu'elle s'est partiellement défaite de son enracinement national et a ainsi rompu les compromis nationaux qui avaient soutenu sa montée en puissance. Dans ce contexte globalisé, la RSE est mobilisée par des hauts managers « comme une garantie face à 'l'apatridisation' de la grande entreprise multinationale " (Capron et Petit, 2011). Conjointement, la RSE est utilisée en vue de renforcer l'attachement des fournisseurs, des salariés, voire des clients. Cet axe stratégique est moins souvent identifié bien qu'il soit cœur de la conception shareholder de l'entreprise, en faisant primer la valorisation des actifs de la firme. Cet objectif conduit les FMN à renforcer leur pouvoir sur leur réseau de sous-traitants. Le développement de la firme en réseau, rendu possible par d'autres facteurs, comme les TIC, constitue un facteur clé de mondialisation de la production et de désintégration verticale de la firme. L'internationalisation des chaînes de valeur dépasse la simple extension de la sous-traitance, allant jusqu'à l'extériorisation complète de la fabrication.

Dans ce cadre, la RSE est utilisée pour suppléer aux défauts de la gouvernance actionnariale. La RSE-Stratégie participe ainsi à la structuration de la gouvernance du réseau d'entreprises dont la coordination est complexe et les conséquences potentiellement coûteuses car elles mettent en jeu la réputation des donneurs d'ordres. Dans les cas Mattel-Barbie (Lamarche et Rubinstein, 2012) et Puma (Palpacuer, 2008) l'atteinte à la réputation de la marque à travers le réseau fournisseur a ainsi des effets sur les stratégies productives. L'appui sur la RSE légitime et normalise les pratiques et les contraintes imposées aux sous-traitants. La firme parvient ainsi à accroître sa flexibilité productive en externalisant largement son activité, tout en développant une gestion du risque contre les pratiques éventuellement contestées de ses sous-traitants.

Loin de porter vers une meilleure répartition du surplus ou vers de nouveaux espaces de délibération dans les filières, la RSE conduit à « un transfert de risque de l'acteur fort vers l'acteur faible dans les CGV» (Vercher et al., 2011). Ces dispositifs aboutissent pour les FMN à une déresponsabilisation au sens juridique. Les codes de conduites procèdent quant à eux par transfert vers le sous-traitant, quand les alertes éthiques transfèrent la responsabilité vers les salariés qui sont incités, voire sommés, de dénoncer des pratiques dont ils dépendent et qui ne relèvent pas de leur fait. Ainsi, il s'agit de «dispositifs qui renforcent les inégalités " (Vercher et al, 2011) et mettent des catégories d'acteurs en position d'assumer un mode d'organisation et des travers qui sont structurels. La logique d'individualisation dans l'entreprise pousse à mettre en cause le salarié en lieu et place du donneur d'ordres.

La multinationalisation de la production est ainsi construite pour reporter la contrainte sur les fournisseurs. Il s'agit d'éviter les réglementations coûteuses (sur l'environnement ou le travail) et contourner l'extension historique des rapports salariaux nationaux, c'est à dire la socialisation de plus en plus poussée de la force de travail (Michel, 2013). L'externalisation engendre une « ineffectivité du droit du travail » (Perraudin et al. 2006) et ralentit ainsi cette tendance historique. En effet les dépenses sociales restent 
considérées par les entreprises en tant que coût à réduire ; la CGV est alors un instrument d'écrasement du rapport salarial.

50 La RSE-Stratégie modifie ainsi les structures de production, engendrant des relations de pouvoirs et des formes de contrôle nouvelles via l'usage de différents instruments qui imposent des règles à intégrer dans la production. L'amélioration de la responsabilité sociale de la firme donneuse d'ordres peut au final aller de pair avec une détérioration sociale chez ses partenaires (Gereffi et al., 2005) du fait de la double contrainte imposée.

\section{Maintien des pouvoirs de la gouvernance actionnariale}

51 La RSE-Stratégie est donc un instrument de discipline au sein des chaînes de valeur mondialisées et instrument de gouvernance inter et intra-firme. Elle confirme l'organisation productive pilotée par la grande firme en lui ajoutant un supplément de légitimité que capte la marque à l'échelle mondiale.

Dès lors que les démarches de RSE-Stratégie sont anticipées en tant que facteurs de valorisation des actifs immatériels, et en dépit de l'absence de preuve, elles sont susceptibles d'intégrer la dynamique actionnariale. Des stratégies de RSE sont intégrées alors aux dispositifs financiarisés, et sont l'objet d'anticipations stratégiques quant à leur rendement. Or dans cette logique de rendement actionnarial, comme l'a décrit Orléan (2011), la valeur qui est en jeu n'est pas «substantielle (ce que montrent les études empiriques); il n'est donc pas nécessaire que ce soit prouvé, ce qui importe, pour la production de valeur actionnariale, est ce que le marché de référence valorise. Le développement de la RSE-Stratégie est à même d'être relayé par une dynamique mimétique propre à la finance.

53 Ce qui est en jeu ici n'est donc pas la remise en cause de l'objectif de maximisation actionnariale, mais la prise de conscience que la poursuite de cet objectif ne peut se faire sans les autres stakeholders et sans des concessions ou des compromis (Jensen 2001). La RSE-Stratégie s'inscrit en tant que réponse stratégique relativement peu coûteuse aux pressions de la critique écologique et sociétale (Miller, 2008). Alors que la RSE-Institution tend à légitimer l'action des entreprises sur le registre politique, la RSE-Stratégie opère, quant à elle, sur le registre productif et de gouvernance, et toujours dans une optique de légitimation. Dans l'esprit des travaux de Fligstein (1990) qui définit les différentes "conceptions du contrôle» dans le sens de représentations de l'entreprise, de ses stratégies et des objectifs qui se sont historiquement succédées, une nouvelle conception émerge, que nous avons désignée " conception shareholder-RSE compatible » (Lamarche et Rubinstein, 2012). Les démarches de RSE-Stratégie, s'appuient dans ce cadre principalement sur une modification de règles privées. Cela confirme l'interaction permanente entre la dynamique des règles et les dynamiques productives. La RSEInstitution permet et stabilise la RSE-Stratégie qui en retour requiert des dispositifs institutionnels qu'elle engendre donc.

L'originalité de l'interaction entre RSE-Institution et RSE-Stratégie provient du processus d'institutionnalisation, marqué par le volontariat et par un mode spécifique d'enforcement . Une des clés de la réussite de la conception shareholder-RSE compatible réside dans ce mouvement de décentralisation de la production des règles: c'est la capacité des entreprises à façonner ces règles, à leur échelle propre ainsi qu'à une échelle collective, qui rend la RSE compatible et cohérente avec la gouvernance actionnariale, tout en palliant certaines faiblesses. 


\section{De la permanence du rapport salarial financiarisé}

55 concurrence et joue ainsi un rôle disciplinaire vis-à-vis du rapport salarial. La domination structurale de la concurrence sur le rapport salarial n'est pas amendée par la RSE qui ne traite que marginalement de répartition et de protection sociale. La RSE n'affecte donc pas le partage inégal salaire / profit propre au régime néolibéral, ni la dynamique de flexibilisation du rapport salarial. La RSE-Stratégie s'ajoute à la gouvernance asymétrique des CGV, elle ne s'y substitue pas. L'intégration d'obligations sociales et environnementales correspond à des enjeux du développement durable, sans remise en cause de concurrence par les prix et de la pression sur les coûts. Il y a là une contraction importante : l'intégration du respect des normes en matière de droit du travail (horaires, salaires, conditions de travail, sécurité) dans les codes de conduite profite marginalement aux salariés. La RSE joue toutefois conjointement comme facteur de mobilisation du travail notamment des cadres des sièges sociaux dans les pays du Nord (Postel et al. 2006).

Le rôle de la RSE se comprend ainsi en rapport à l'évolution du rapport salarial. La financiarisation instrumente la déstabilisation permanente du rapport salarial pour dégager des marges nouvelles. Cette déstabilisation est cumulative du fait de la mise en concurrence des rapports salariaux nationaux. Ainsi, déstabilisé structurellement le rapport salarial n'est plus le soutien premier à l'accumulation, il perd sa centralité dans le régime (Michel 2013) ; il n'est plus la forme institutionnelle structurant le bouclage de la demande (Montagne 2006). Plus instable et individualisé, le rapport salarial financiarisé est reconfiguré de façon à apporter plus de flexibilité dans la production. Et le développement de la RSE-Stratégie n'infléchit en rien cela, car c'est un facteur de compétitivité supplémentaire.

Avec l'emboîtement des réseaux de fournisseurs qui se développe dans les CGV, la maind'œuvre est mobilisée en dehors d'un contrat de travail sur lequel le donneur d'ordre n'est donc pas formellement impliqué (Supiot 2011). Cela traduit une déresponsabilisation des FMN à l'égard du salariat. Leur gouvernance est construite dans mise à distance des travailleurs et du donneur d'ordres, qui est employeur de fait, mais non de droit ${ }^{22}$. Ainsi le rapport salarial est contourné par ces dispositifs d'organisation et de production à l'échelle mondiale. La main-d'œuvre est mobilisée hors de tout contrat de travail avec le donneur d'ordres et des régimes de protection juridique y afférant. Les relations ne sont plus régulées par le droit social et le droit du travail, mais par d'autres systèmes de droit, déplaçant le droit social vers la lex mercatoria (Bodet et Lamarche 2007). La relation avec les syndicats est ainsi partiellement contournée, et combattue à l'échelle locale, elle revient toutefois à une échelle globale (ISO 26000, Accords Cadres Internationaux $\left.{ }^{23} . ..\right)$. En horizontalisant tout un ensemble d'enjeux (sociaux, environnementaux, droits humains, bien-être au travail, etc.), la RSE joue de façon ambivalente sans offrir d'institutionnalisation du rapport salarial à une échelle internationale. Dans ce sens la RSE participe d'une désinstitutionnalisation du rapport salarial.

Par l'intermédiaire des codes et chartes intégrés dans les chaînes de valeur, les FMN disposent d'instruments qui s'ajoutent à une gouvernance en réseau. Ces instruments n'agissent cependant pas à une échelle microéconomique. La capacité de contrôle que développent les FMN assure à une échelle mésoéconomique une compatibilité dynamique 
d'un ensemble de décisions décentralisées, n'agissant pas toutefois sur la régulation d'ensemble.

Des éléments de régulation sont donc à l'œuvre, à l'échelle des FMN notamment: les dispositifs de RSE mobilisés afin de répondre à la critique sociale aboutissent, compte tenu des rapports de force en présence, à renforcer le contrôle sur les fournisseurs. Cela n'atténue pas la logique polarisée et oligopolistique, cependant les dispositifs RSE participent à une forme de régulation des rapports contradictoires, entre firmes, mais aussi entre capital et travail à l'échelle internationale. Cette coordination est reléguée toutefois au niveau de la production et n'assure pas de stabilisation macroéconomique. Il s'agit d'une régulation qui se présente ainsi essentiellement à l'échelle mésoéconomique des CGV et des grandes firmes.

\subsection{Variété des conventions de qualité et avantages concurrentiels : une RSE sans montée en régime}

\section{Vers une introuvable convention de qualité ?}

60 À la dynamique amont des CGV est associée une dynamique aval en lien étroit avec l'émergence de nouvelles conventions de qualité intégrant la responsabilité, nous les appelons conventions de 'qualité responsable'. L'économie des conventions retient la qualité en tant que critère conventionnel identitaire (quelle valeur caractérise une qualité donnée ?), la normalisation intervenant en tant qu'épreuve de conformité (quel processus permet de valider une qualité attendue ?) (Eymard-Duvernay, 1989). Une convention de qualité ne s'instaure toutefois que si elle assure une rentabilité pour le producteur, en quelque sorte si elle correspond à l'intérêt que lui portent les groupes fondateurs. C'est précisément ce qui est en jeu avec la RSE-Stratégie. En la matière, il s'agit des produits labellisés équitables, responsables, solidaires, et biologiques qui répondent à différentes formes de consommation engagée (Dubuisson-Quellier, 2009) et ont trouvé des débouchés hors du champ alternatif d'origine.

61 Ce processus suppose que ces conventions soient reconnues des consommateurs. À l'échelle mésoéconomique des grandes firmes, se construisent ainsi des normes de qualité, elles sont de puissances et de contenus variés, et sont en concurrence. Ces conventions agissent en tant qu'institution. Leur principe d'action est un pouvoir symbolique sur les représentations sociales des individus, qui mobilise des dimensions matérielles et idéelles. La RSE-Stratégie se traduit à l'échelle des entreprises par une pluralité de dispositifs de valorisation des produits à partir de qualités multiples ayant trait à des aspects environnementaux, sociaux ou territoriaux. Les qualités responsables sont mobilisées dans la dynamique concurrentielle en même temps que d'autres attributs qui ne sont pas compatibles (innovation, prix) ${ }^{24}$.

Actuellement, les conditions de marché ne sont pas réunies pour imposer une norme de concurrence structurée par une convention de 'qualité responsable' dominante, quelle que soit sa définition car le coût de production est trop important. Il y a bien foisonnement d'initiatives en la matière, mais pas de convention structurante. La norme ISO 26000 ne parvient pas à jouer le rôle escompté de convention de qualité internationalement reconnue. Le développement de dispositifs de management de la qualité est une manière pour le management interne de défendre une marge de manœuvre face aux contraintes actionnariales. Cependant les conditions de la production 
d'une 'qualité responsable' se situent nécessairement dans des rapports sociaux (rapport salarial, rapport aux populations locales, rapport aux ressources et à l'environnement), or la dynamique de RSE-Stratégie ne traite pas de ces effets externes. Elle reste soumise à la concurrence internationale dans laquelle les prix sont structurants et ne permettent pas l'intégration des externalités.

La dynamique de RSE ne constitue pas un moment stable de la régulation du capitalisme, mais au contraire un moment transitionnel, durant lequel émergent de nouvelles conventions, associées à l'évolution des modes de consommation. Cette évolution est toutefois conditionnée par la répartition, qui reste fondamentalement inégalitaire.

\section{Un régime de demande incompatible avec la « qualité responsable »}

La dynamique néolibérale repose sur un conflit de répartition largement dominé par le capital. L'évolution du partage de la valeur ajoutée sur longue période, montre que dans tous les pays développés la part des salaires baisse (de 8 points dans l'Union européenne de 1961 à 2009) et respectivement la part des profits s'accroît ; dans la plupart des pays développés, les dividendes versés s'accroissent à mesure que le taux d'investissement chute (Husson, 2010). Or les dispositifs RSE ne traitent aucunement de ce point crucial qui reste une limite à la reproduction du régime financiarisé et accroît sa fragilité (Setterfield, 2011). Sur le plan macroéconomique la généralisation d'une logique de responsabilité sociale depuis les CGV jusqu'à une régulation d'ensemble est ainsi hypothéquée par un niveau de demande dépressif. La pression sur le pouvoir d'achat des salariés au Nord n'offre pas de débouché à la convention de 'qualité responsable'. Pour l'essentiel, les régimes de demande des pays du Sud, quant à eux, ne sont pas tournés vers des marchés intégrant ces conventions de qualité, mais sont tirés par l'émergence d'une classe moyenne portée vers les premiers équipements et une qualité relevant du registre industriel. Dans un tel régime de demande atone, les formes de concurrence restent donc contraintes par les prix.

Si la RSE s'intègre aux modes de coordination et à l'organisation de la production, qui composent l'accumulation, il n'y a pas de modification substantielle du régime d'accumulation, ni même d'annonce d'un régime préfigurant une sortie de crise. Le processus d'institutionnalisation de la RSE est signifiant des rapports de pouvoir économique et renvoie à la tension entre le pouvoir réglementaire et l'éclosion de dispositifs unilatéraux engendrés par les dispositifs stratégiques qui, en l'état, ne produisent pas de norme stable. Les normes qui émergent dans la RSE-Stratégie sont en effet fortement réversibles puisque sujettes à remise en cause au gré de l'évolution des rapports de concurrence. La nature et l'étendue de l'information nécessaire à l'élaboration d'une convention de qualité responsable tout au long d'une filière est d'une telle complexité qu'elle n'est de fait possible que pour le management interne. La maîtrise des CGV est donc le lieu du pouvoir managérial, et les questions, complexes et variées, traitées dans la RSE-Stratégie sont en mesure de renforcer ce pouvoir interne. La maîtrise de l'information agit en tant que rente pour ceux qui la détiennent (Rebérioux, 2007), ce qui renvoie à la position pivot des hauts dirigeants. Intégrée dans les dispositifs institutionnels de la finance et de la concurrence internationale, la RSE-Stratégie joue de fait dans le sens du renforcement du mode de régulation financiarisé, plutôt que dans son atténuation, mais fait évoluer les institutions de la de la RSE au sein des CGV. 


\section{Conclusion}

Sans dispositifs de valorisation, pas de montée en régime de la RSE

Les processus de normalisation transforment progressivement, et en partie, l'organisation productive à l'échelle mésoéconomique, de certains secteurs (alimentation (Jones et Nisbet, 2011), chaussure (Palpacuer 2008), Télécom (Rubinstein et Cézanne, 2012), etc.). Pour autant, à défaut d'une norme à caractère général, envisager un mode de régulation dans lequel la RSE joue un rôle central au sein des rapports sociaux et soutenant un régime macroéconomique stable apparait hors de portée. Le niveau pertinent de création de normes est l'échelle intermédiaire, notamment sectorielle, mais aussi celle des CGV, des firmes. Notons bien la contradiction apparente : la RSE interpelle le niveau entreprise, elle est l'objet d'une intense activité de la part des grandes entreprises à propos de sa définition et de son mode opératoire (i.e. volontariat et absence de coercition); pourtant les faits stylisés RSE-Institution et RSE-Stratégie ne relèvent pas du niveau de l'entreprise, mais d'un niveau de généralité qui les englobe, alors même que leur intention de départ est de maîtriser ces normes. C'est un point de contradiction propre aux processus d'institutionnalisation.

Les conventions de qualité qui émergent peuvent s'imposer localement ou dans des soussecteurs, mais ne fondent pas un mode de valorisation du capital à même de contrecarrer la dynamique financiarisée. En effet, ce qui n'est pas directement mesuré selon les critères de l'évaluation financière est ignoré par les dispositifs du régime d'accumulation qui définissent ce qui a de la valeur (i.e. les normes de rendement). Ainsi le respect des principes (égalité femmes hommes, respect des patrimoines naturels, processus de décision démocratique, etc.) n'entre pas dans un tel mode de valorisation. Dans une phase de crise structurelle, la RSE offre conjointement de la continuité (reproduction du régime) et des ferments de transformation sociale (conventions de qualité, accordscadres internationaux, codes de conduite). Cependant, les dispositifs de RSE sont peu saisis dans les rapports sociaux et notamment dans les rapports capital-travail : ils restent l'apanage de nouvelles parties prenantes (particulièrement des $\mathrm{ONG}$ ), qui sont faibles au regard des FMN et peu instituées, donc sans pouvoir structurant. Dans le même temps, les représentants du travail sont tenus à distance de la RSE qui reste un objet des directions d'entreprise. Or les hauts dirigeants ont structurellement noué un compromis avec la finance de marché par l'intermédiaire de rémunérations actionnariales (Duménil et Lévy, 2011). Pendant que le compromis social institutionnalisé exploite l'érosion du pouvoir de négociation des fournisseurs et des salariés, les dispositifs de RSE ne traitent pas de cette dimension structurelle. Le développement durable formulé par la RSE échoue sur le volet social en ce qu'il y a régression dans les modalités du rapport salarial.

La tension mise en avant entre le politique et le productif (entre RSE-Institution / RSEStratégie) rend compte de la façon dont se nouent les contractions au sein de la grande entreprise. L'écrasement du social, alors même que des stratégies RSE sont mobilisées, annonce d'amples difficultés. Il nous semble important mettre cela en résonnance avec les perspectives de recomposition / refondation de l'entreprise en tant qu'institution (Hatchuel et Segrestin, 2012, Favereau, 2012). En effet la nature de l'activité, au sens du modèle économique susceptible d'engendrer des profits, ne saurait être durablement coupée du compromis social au sein de l'entreprise. 


\section{BIBLIOGRAPHIE}

Acquier, A. et Aggeri, F., (2008), "Entrepreneuriat institutionnel et apprentissages collectifs. Le cas de la Global reporting initiative (GRI)", Management international 12-2, 65-80.

Aglietta, M. (1998), « Le Capitalisme de demain », Notes de la fondation Saint-Simon, n 101, novembre.

Aglietta, M. et Rebérioux, A., (2004), Dérives du capitalisme financier, Albin Michel, Paris.

André, R. et Delorme, C., (1983), L'État et l'économie, Seuil, Paris.

Barnejee, S.B. (2011), "Voices of the governed: towards a theory of the translocal", Organisation, 18 (3), 323-344.

Blair, M., (2000), The new relationship: human capital in the American corporation, Brooking Institution press, Washington.

Bodet, C. et Lamarche, T., (2007), "La responsabilité sociale des entreprises comme innovation institutionnelle. Une lecture régulationniste", Revue de la Régulation,

http://regulation.revues.org/1283

Boltanski, L. et Chiapello, E., (1999), Le nouvel esprit du capitalisme, Gallimard, Paris.

Boyer, R. (1986), La théorie de la régulation une analyse critique, La Découverte, Paris.

Boyer, R, (1992), «La crise de la macroéconomie, une conséquence de la méconnaissance des institutions ?", L'Actualité économique, vol. 68, n 1-2, 1992, p. 43-68.

Boyer, R., (2004), Théorie de la régulation. 1. Les fondamentaux, Repères, La découverte, Paris.

Boyer, R., (2005), "From Shareholder Value to CEO Power: The Paradox of 1990's", Competition \& Change 9-1, 7-47.

Boyer, R. et Saillard, Y. (ed.), (2002), Théorie de la régulation, L'état des savoirs, La découverte, Paris. de la Broise P. et Huet R. (2010), « La responsabilité sociale des entreprises en quête d'auteurs », Communication et organisation, 37, pp. 65-77.

de la Broise, P. et Lamarche, T. (2006), Responsabilité sociale : vers une nouvelle communication des entreprises?, Septentrion, Lille.

Capron, M., (2006), "Responsabilité d'entreprise : le renversement européen", Bulletin Oeconomia Humana 4,3 .

Capron, M. et Petit, P., (2011), «RSE et diversité des capitalismes dans la phase contemporaine d'internationalisation ", Revue de la Régulation,

http://regulation.revues.org/9085

Capron, M. et Quairel-Lanoizelée, F., (2004), Mythes et réalités de l'entreprise responsable, La Découverte, Paris.

Capron, M., Quairel-Lanoizelée, F. et Turcotte, M.-F., (2011), ISO 26000 : une Norme "hors norme" ?, Economica, Paris.

Cézanne, C., et Rubinstein, M. (2012), « La RSE comme instrument de gouvernance d'entreprise : une application à l'industrie française des télécommunications », Revue de la régulation $12,2 \mathrm{e}$ 
semestre / Autumn,

http://regulation.revues.org/9819

Chandler A. D. Jr. (1990), Scale and Scope: The Dynamics of Industrial Capitalism, Cambridge: Belknap Press of Harvard University Press.

Chanteau, J.-P. (2011), « L'économie de la responsabilité sociétale d'entreprise : éléments de méthode institutionnaliste ", Revue de la régulation $\mathrm{n}^{\circ} 9$, 1er semestre / Spring http://regulation.revues.org/9328

Chanteau, J.-P. et Lamarche, T., (2013) « Modes de régulation », in Postel, N. et Sobel, R., (ed), Dictionnaire critique de la RSE, Septentrion, pp. 311-316.

Chassagnon, V., (2011), « The Law and Economics of the Modern Firm: A New Governance Structure of Power Relationships ». Revue d'Économie Industrielle, n 134, p. 25-50.

Chassagnon, V., (2013), « Contrôle et manipulation au cœur de la firme-monde ? », Revue de la Régulation, http://regulation.revues.org $n^{\circ} 14$, Autumn.

Chiapello E. (2013), « Capitalism and its criticisms », in G. Morgan and P. du Gay (eds), New spirits of capitalisms, Oxford University Press.

Clévenot, M., (2008), " Les difficultés à nommer le nouveau régime de croissance », Revue de la régulation, $\mathrm{n}^{\circ} 3 / 4,2^{\mathrm{e}}$ semestre / Autumn.

http://regulation.revues.org/7606

Clos, C. (2013), « Les processus d'institutionnalisation de la RSE : l'exemple des lois de Reporting en France. Etude du processus de construction et d'application de l'article 225 de la loi Grenelle II de l'Environnement », Colloque RIODD, Lille, 18-21 Juin.

Collins, H., (1990), « Ascription of Legal Personality to Groups in Complex Patterns of Economic Integration ». The Modern Law Review, vol. 53, nº 6, p. 731-744.

Courent, J-M (2012), RSE et développement durable en PME, De Boeck.

Dannreuther, C. and Petit, P. (2012), "Introduction to the special issue on the regulation approach and the contemporary crisis", Capital \& Class, 37(1) 3-4, pp. 3-4.

Descolonges, M., (2006), Implications for French Unions involved in International Framework Agreements , International conference "Global Companies-Global Unions-Global Research-Global Campaigns, New York.

Descolonges, M. et Saincy, B., (2004), Les entreprises seront-elles un jour responsables ?, La dispute, Paris.

Desmoulins, L. et Lamarche, T., (2013) « Des think tanks producteurs de 'valeurs publiques' et de légitimités privées : discours et stratégies de communication sur la RSE », in Bournois, F., Chanut, V. et Rival, V., Intelligence économique et lobbying, Ed ESKA

Dubuisson-Quellier, S. (2009), La consommation engagée, Ed. Presses de Sciences Po, 2009.

Duménil G., Lévy D. (2011), The Crisis of Neoliberalism, Harvard University Press, Cambridge MA.

Dupuis, J.-C., (2008), "La responsabilité sociale de l'entreprise : gouvernance partenariale de la firme ou gouvernance de réseau ?", Revue d'économie industrielle n 122, pp. 67-86.

Eymard-Duvernay, F. (1989), « Conventions de qualité et formes de coordination », Revue économique, $\mathrm{n}^{\circ} 2$, mars, pp. 329-359. 
Favereau, O., (2012), « Investigations et Implications : synthèse des travaux », in Roger, B. et al, L'entreprise, formes de la propriété et responsabilités sociales, Edition Lethiellieux - Collège des bernardins, pp. 17-103.

Favereau O. (2013), « Pour un nouveau modèle d'entreprise », Rapport moral sur l'argent, Association d'économie financière.

Fligstein, N., (1990), The transformation of corporate control, Harvard University Press, Cambridge.

Gallois F. et Nieddu M. (2015), « Quand l'État décrète le marché : le cas du Plan Borloo », Revue de la régulation 17 | 1er semestre / Spring 2015, http://regulation.revues.org/11209

Gereffi, G., Humphrey, J., Sturgeon, T. (2005), "The Governance of Global Value Chains", Review of International Political Economy, vol. 12, No. 1, February, pp. 78-104.

Girard, B. (2011), « Les Nouveaux Mouvements Socio-Economiques au regard de l'histoire », Revue de l'organisation responsable, vol. 6-1.

Hatchuel, A., et Segrestin, B. (2012) Refonder l'entreprise, Seuil, La république des idées.

Hoang-Ngoc, L. et Tinel, B., (2005), « La régulation du 'nouveau capitalisme'. Analyses positives et recommandations normatives comparées », Economie Appliquée, LVIII - 1, pp. 33-57.

Husson, M., (2010), « Le partage de la valeur ajoutée en Europe », La revue de l'IRES Nº64, pp. 47-92.

Jensen M.C. (2001), "Value Maximisation, Stakeholder Theory, and the Corporate Objective Function", European Financial Management, vol. 7 (3), pp. 297-317.

Jeammaud, A., (2000), «L'interdisciplinarité, épreuve et stimulant pour une théorie des règles juridiques », in Kirat, T. et Serverin, E., Le droit dans l'action économique, CNRS éditions, Paris.

Jones, B., et Nisbet, P, (2011), « A Better Model for Socio-economic Governance ? », Revue de la régulation, 9 1er semestre / Spring 2011, http://regulation.revues.org/9158

Labrousse, E. (1944), La crise de l'économie française à la fin de l'Ancien Régime et au début de la Révolution, PUF.

Lafaye de Micheaux E. et Alary P. (2013), « L'économie politique de l'Asie : état des lieux et perspectives de recherche pour l'Asie du Sud-Est », Revue de la régulation, 13, Spring.

Lamarche, T., (2009), "Les nouvelles institutions de la 'mesure' de la RSE entre communication et évaluation", Revue de l'organisation responsable $\mathrm{n}^{\circ} 2$ vol. 4, 4-18.

Lamarche, T. (2011) « Responsabilité sociale des entreprises, régulation et diversité du capitalisme ", Revue de la Régulation, $\mathrm{n}^{\circ}$ 9, 1er sem., http://regulation.revues.org/9275

Lamarche, T. and Rubinstein, M., (2012), "Dynamics of corporate social responsibility: towards a new 'conception of control'?", Journal Of Institutional Economics, Issue 8(2), June 2012.

Lamarche, T., Nieddu, M.,Grouiez, P., Chanteau, J-P, Labrousse, A., Michel, S.et Vercueil, J. (2015), "Les régulations mésoéconomiques : saisir la variété des espaces de régulation", Colloque La théorie de la régulation à l'épreuve des crises Paris, France. p. 1-23. <hal-01163875>

Lordon, F., (1999), «Le nouvel agenda de la politique économique en régime d'accumulation financiarisé », in Duménil, G. and Lévy, D. (éd.). Le Triangle infernal : Crise, mondialisation, financiarisation, Paris, PUF. 
Lordon, F. (Ed.), (2008), Conflits et pouvoirs dans les institutions du capitalisme, Presses de SciencesPo, Paris.

Margolis J., Elfenbein H. A. (2008) “Doing well by doing good? Don't count on it”, Harvard Business Review, vol. 86, $\mathrm{n}^{\circ} 1$, p. 19-20.

Martinez-Alier J., Pascual U., Vivien F.D. and E. Zaccai, (2010) "Sustainable de-growth: Mapping the context, criticisms and future prospects of an emergent paradigm", Ecological Economics, 69 1741-1747.

Michalet, C.-A., (1985), Le capitalisme mondial, PUF, Paris.

Michel, S., (2013), "Wage Form and Social Spending in the Long Run", EAEPE Annual Conference Paris Nord/13 University, November 7-9.:

Miller, J., (2008), "The ongoing legitimacy project: corporate philanthropy as protective strategy", European Management Review, n 5, 151-164.

Montagne, S., (2006), Les fonds de pension. Entre protection sociale et spéculation financière, Odile Jacob, Paris.

Orléan, A., (2009), De l'euphorie à la panique : Penser la crise financière, éd. de la Rue d'Ulm.

Orléan, A., (2011), L'empire de la valeur. Refonder l'économie, Le Seuil, Paris.

Orlitzki, M., Schmidt, F. L. et Rynes, S. L., (2003), "Corporate social and financial performance: a meta-analysis", Organization Studies 24 (3), 403-441.

Palpacuer, F., (2008), "Firme-réseau globale et réseaux transnationaux d'ONG : Vers un nouveau mode de régulation ?" Revue de la Régulation, $\mathrm{n}^{\circ} 2$.

http://regulation.revues.org/2243

Penalva Icher E. (2009), « Construire une qualité pour le 'socialement responsable' ? », Revue Française de Socio-Économie, $n^{\circ} 2-4,59-81$

Perraudin C., Thévenot N., Tinel B. et Valentin J. (2006), « Sous-traitance dans l'industrie et ineffectivité du droit du travail : une analyse économique », Economie et institutions, 35-55.

Plihon, D., (2009), Le nouveau capitalisme, Repères-La découverte, Paris.

Porter, M. and Kramer, M., (2006), "Strategy \& Society: the link between competitive advantage and corporate social responsibility", Harvard Business Review Spotlight.

Postel, N., Rousseau, S. et Sobel, R., (2006), « La 'responsabilité sociale et environnementale des entreprises' : une reconfiguration potentielle du rapport salarial fordiste? » Économie appliquée LIX, 77-104.

Rebérioux, A., (2007), "Does shareholder primacy lead to a decline in managerial accountability?" Cambridge Journal of Economics 31, 507-524.

Roger, B. et al (2012), L'entreprise, formes de la propriété et responsabilités sociales, Édition Lethiellieux - Collège des bernardins.

Rousseau, S. et Zuindeau, B., (2007), "Théorie de la régulation et développement durable", Revue de la Régulation,

http://regulation.revues.org/1298

Setterfield, M., (2011), « Anticipations of the Crisis: On the Similarities between post-Keynesian Economics and Regulation Theory ", Revue de la régulation, 10, 2e semestre / Autumn, http://regulation.revues.org/9366 
Supiot, A., (2010), L'esprit de Philadelphie : la justice sociale face au marché total, Éditions du Seuil, Paris.

Théret, B. dir, (2008), La monnaie dévoilée par ses crises, Edition de l'EHESS.

Vercher, C., Palpacuer, F., et Charreire Petit, S., (2011), « Codes de conduite et systèmes d'alerte éthique : La RSE au sein des chaînes globales de valeur », Revue de la régulation, 9, 1er semestre / Spring,

http://regulation.revues.org/9259

Weinstein, O., (2012), "Firm, Property and Governance: From Berle and Means to the Agency Theory, and Beyond", Accounting, Economics, and Law. A Convivium, vol. 2 (2).

\section{NOTES}

1. Voir ainsi Novethic expliquant que « la notion de RSE est liée à l'application aux entreprises du concept de développement durable » (http://www.novethic.fr).

2. La mobilisation d'acteurs collectifs est ainsi essentielle, voire notamment leur caractérisation en termes d'entrepreneurs institutionnels, Acquier et Aggeri, 2008.

3. Codifications que la théorie de la régulation désigne en termes de « Formes institutionnelles ».

4. Sur la RSE dans les PME, voir Courent, 2012.

5. Kaldor, N. (1961), Economic growth and capital accumulation, in F. Lutz et D. C. Hague, eds, "The Theory of Capital", Macmillan, London, pp. 177-222. Sur la centralité des faits stylisés et le recours aux faits stylisés paradoxaux voir aussi Gallois et Nieddu, 2015.

6. La grille repose sur une activité d'évaluation de la RSE et d'accompagnement d'entreprises, mené par une des auteures, Catherine Bodet, au sein de différents organismes professionnels.

7. A propos des différentes désignations de ce régime, et des controverses qui y sont liées, voir Clévenot, 2008.

8. Pour des développements sur la notion de « Nouveaux mouvements socio-économiques », voir Girard, 2011.

9. Voir, Les échos, 21 juin 1995 ou les commentaires de Christian Harbulot sur son blog http:// www.infoguerre.fr/guerre-de-l-information/greenpeace-contre-shell/

10. Voir le site du comité de coordination pour le Rana Plaza http://www.ranaplazaarrangement.org/

11. Ce que Théret a mis au cœur des travaux sur la monnaie avec un titre qui pose la crise comme révélateur : «La monnaie dévoilée par ses crises ».

12. Cf. La doctrine défendue par Friedman, "The Social Responsibility of Business is to Increase its Profits", The New York Times Magazine, 13/09/1970.

13. Voir ainsi comment deux think tanks français parmi les plus actifs (Terra Nova et l'Institut Montaigne) traitent massivement de thématiques liées à la RSE (Desmoulins et Lamarche, 2013).

14. EMAS, aussi désigné éco-audit, provient d'un règlement européen de 1995 qui a pour objet de cadrer les démarches volontaires d'écomanagement.

15. L'action des grands cabinets de conseil à ce sujet est tout à fait notable (Clos, 2013).

16. Voir les travaux sur l'émergence de dispositifs professionnels à propos de la RSE, qui mènent à une dynamique de professionnalisation (de la Broise et Huet, 2010).

17. Cela correspond à la définition que Lordon donne des « institutions de pouvoir » (2008).

18. L'économie politique de l'Asie nous montre ainsi une variété de situations et de rôles possible des Etats qui illustrent parfaitement la tension entre un processus d'homogénéisation qu'engendrent les normes de concurrence à l'échelle mondiale et la permanence d'une diversité d'espaces locaux (Lafaye de Micheaux et Alary, 2013). 
19. Le Pacte de responsabilité initié fin 2013 en France est un exemple de cet esprit de la RSE. Il est fondé selon François Hollande «sur le principe simple : moins de charges sur le travail, moins de contraintes sur leurs activités et, en même temps, une contrepartie, plus d'embauches et plus de dialogue social ». L'allègement d'un certain nombre de règles sociales ne s'accompagne toutefois d'aucune obligation formelle imposée aux entreprises.

20. Sur le rôle de l'incertitude radicale dans la crise, voir Orléan 2009.

21. A la suite des travaux d'Aglietta et Rebérioux (2004) caractérisant le rôle des intermédiaires (gate keepers) dans les dérives du capitalisme financier, on a montré comment se structuraient les gate keepers de la RSE, eux aussi sous influence (Lamarche, 2009).

22. C'est le cas de nombreux groupes qui sont devenus «fabless » et ne sont plus responsables au premier rang de la relation salariale pour la production matérielle. Voir notamment les affaires autour des conditions de travail, du travail forcé et des suicides dans les usines Foxconn (notamment auprès de Human Right Watch).

23. Les ACI sont constitutives d'un dialogue social international et peuvent préfigurer des conventions collectives à cette échelle (en replis pour les pays du nord, en progrès pour d'autres) (Descolonges, 2006).

24. Voir dans ce sens les travaux sur les conventions de qualité concurrentes en matière d'Investissement Socialement Responsable (Penalva Icher, 2009).

\section{RÉSUMÉS}

La RSE correspond à de deux faits stylisés distincts que nous analysons en termes de régulation et de pouvoir. Celui qui prime est de nature institutionnelle et politique, nous le nommons RSEInstitution, il rend possible et stabilise le second, que nous nommons RSE-Stratégie, de nature économique, productive qui en retour engendre et requiert des compromis politiques que structurent les dispositifs institutionnels. Les instruments de gouvernance interfirme au sein des chaînes globales de valeur, et l'émergence des conventions de "qualité responsable" ne permettent pas toutefois le dépassement du régime financiarisé, mais tendent au contraire à le renforcer, notamment $\mathrm{du}$ fait du contournement du rapport salarial.

CSR reflects two distinct stylised facts that we analyse in stake of regulation and power. The first is of institutional and political nature, we call it institutional-CSR. It Allows and stabilises the second one, which is of economic and productive nature, that we call Strategic-CSR. The Strategic-CSR makes possible and in the same time requires some political compromises structured by institutional devices. The instruments of interfirm governance within global values chains, and the emergence of "quality responsible" conventions do not led to overtake the financialised regime. However quite the contrary they tend to reinforce it, mainly through bypassing wage-labor nexus. 
INDEX

Mots-clés : RSE, institution, régulation, chaînes globales de valeur, convention de qualité

Keywords : CSR, institution, regulation, global value chains, quality convention

Code JEL D2 - Production and Organizations, B52 - Institutional • Evolutionary, L15 -

Information and Product Quality • Standardization and Compatibility, M14 - Corporate Culture • Diversity $•$ Social Responsibility, P17 - Performance and Prospects

\section{AUTEURS}

\section{CATHERINE BODET}

Coordinatrice de la commission recherche, Coopaname, 3-7 rue Albert Marquet, 75020 PARIS France

catherine.bodet[at]ouvaton.org

\section{THOMAS LAMARCHE}

MCF HDR Université Paris Diderot - UMR LADYSS CNRS, 5 Rue Thomas Mann, 75013 Paris -

France

Corresponding author: lamarche[at]univ-paris-diderot.fr 